International Journal of Biology, Pharmacy and Allied Sciences (IJBPAS) 'A Bridge Betueen Caboratory and Q Qnendo'

WWW.iibpas.com

\title{
A REVIEW ON ANALYTICAL METHODS FOR ESTIMATION OF TELMISARTAN IN PHARMACEUTICALS
}

\section{KALPANA P*, JAMEELA SK, PRACHET P AND RAMA RAO N}

Department of Pharmaceutical Analysis, Chalapathi Institute of Pharmaceutical Sciences,

Chalapathi Nagar, Lam, Guntur-522034

*Corresponding Author: Kalpana Porumalla: E mail: porumallakalpana@gmail.com

Received $29^{\text {th }}$ Sept. 2020; Revised 25 ${ }^{\text {th }}$ Oct. 2020; Accepted $16^{\text {th }}$ Nov. 2020; Available online $1^{\text {st }}$ Sept. 2021

\section{https://doi.org/10.31032/IJBPAS/2021/10.9.5616}

\section{ABSTRACT}

The present review focuses on estimation of Telmisartan and its combination of drugs by different analytical techniques. Telmisartan is very effectively used in the treatment of hypertension. It is a potent inhibitor of binding of angiotensin-II to the angiotensin -II $\mathrm{AT}_{1}$ receptor and blocks the secretory effects of angiotensin-II and thus reduces hypertension. This review discusses the analytical methods such as UV-spectroscopy, High performance liquid chromatography (HPLC), High performance thin layer chromatography (HPTLC) for the estimation of Telmisartan in bulk and pharmaceutical dosage forms in various research publications. This review enlightens the various solvents and conditions suitable for the estimation of Telmisartan and its combination of drugs. The techniques illustrated here may find application in the qualitative and quantitative estimation of Telmisartan and in analyzing other related properties.

Keywords: Telmisartan, Antihypertensive agent, UV- Spectroscopy, HPLC, HPTLC \section{INTRODUCTION}

Hypertension is one of the most common chronic disorders characterized by elevated blood pressures [1]. Telmisartan is an Angiotensin-II receptor antagonist (ARB) used in the treatment of hypertension in single or in combination with other classes of antihypertensives, also used in treating patients with diabetic nephropathy and in cases of congestive heart failure. Telmisartan, an Angiotensin-II receptor blocker bind to the angiotensin-II type 1 receptors with high affinity, leading to the inhibition of the action of angiotensin II on vascular smooth muscle, eventually causing 
a reduction in arterial blood pressure [2].

Chemically Telmisartan is '2-[4-[[4-methyl-

6-(1-methylbenzimidazol-2-yl)-2-

propylbenzimidazol-1-

yl]methyl]phenyl]benzoic acid. It has a molecular formula of $\mathrm{C}_{33} \mathrm{H}_{30} \mathrm{~N}_{4} \mathrm{O}_{2}$ with molecular weight $514.6 \mathrm{~g} / \mathrm{mol}$. It has a melting point of $261^{0}-263^{\circ} \mathrm{C}$. It is insoluble in water and is sparingly soluble in strong acid and soluble in strong base [3]. Because of its therapeutical importance, the quantitative estimation of Telmisartan has considerable significance in pharmaceuticals. This article reviews analytical methods are developed for the estimation of Telmisartan in bulk and in combination with other drugs.

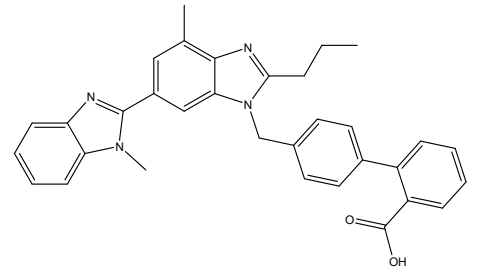

Figure 1: Structure of Telmisartan METHODS FOR TELMISARTAN

UV Spectrophotometric methods:

Various methods for the estimation of Telmisartan in single and in combination with other drugs by UV Spectroscopic methods developed are enlisted in the Table 1.

\section{Chromatographic methods:}

Several chromatographic methods like HPLC, HPTLC and UPLC were developed for estimation of Telmisartan in single and combination with other drugs. Methods for estimation of Telmisartan by HPLC and HPTLC are enlisted in the Table 2, 3.

Table 1: Methods for the estimation of Telmisartan in single and in combination with other drugs by UV

\begin{tabular}{|c|c|c|c|c|}
\hline \multicolumn{5}{|c|}{ Spectroscopy } \\
\hline S. No. & DRUGS & APPLICATION & DESCRIPTION & REF NO \\
\hline 01 & Telmisartan & $\begin{array}{c}\text { Bulk and } \\
\text { pharmaceutical } \\
\text { dosage form }\end{array}$ & $\begin{array}{c}\text { Detection wave length: } 249 \mathrm{~nm} \\
\text { Solvent: Methanol } \\
\text { Linearity range: } 5-100 \mu \mathrm{g} / \mathrm{ml} \\
\text { Correlation coefficient: } 0.999\end{array}$ & [4] \\
\hline 02 & $\begin{array}{c}\text { Telmisartan and } \\
\text { Metoprolol succinate }\end{array}$ & $\begin{array}{l}\text { Combined tablet } \\
\text { dosage form }\end{array}$ & $\begin{array}{c}\text { Detection wave length: } \\
\text { Telmisartan: } 296 \mathrm{~nm} \\
\text { Metoprolol succinate: } 223 \mathrm{~nm} \\
\text { Solvent: Methanol } \\
\text { Linearity range: } \\
\text { Telmisartan: } 2-16 \mu \mathrm{g} / \mathrm{ml} \\
\text { Metoprolol succinate: } 3-24 \mu \mathrm{g} / \mathrm{ml} \\
\end{array}$ & [5] \\
\hline 03 & Telmisartan & $\begin{array}{c}\text { Bulk and tablet } \\
\text { dosage Form }\end{array}$ & $\begin{array}{c}\text { Detection wave length: } \\
\text { Zero order }: 235 \mathrm{~nm} \\
\text { Solvent: } 0.1 \mathrm{~N} \text { NaOH } \\
\text { Linearity range: } 4-25 \mu \mathrm{g} / \mathrm{ml} \\
\text { Correlation coefficient: } 0.999\end{array}$ & [6] \\
\hline 04 & $\begin{array}{c}\text { Telmisartan } \\
\text { Hydrochlorthiazide and } \\
\text { Amlodipine Besylate }\end{array}$ & $\begin{array}{l}\text { Bulk and combined } \\
\text { tablet dosage form }\end{array}$ & $\begin{array}{c}\text { Detection wave length: } \\
\text { Telmisartan: } 296 \mathrm{~nm} \\
\text { Hydrochlorthiazide: } 272 \mathrm{~nm} \\
\text { Amlodipine Besylate: } 360 \mathrm{~nm} \\
\text { Solvent: Methanol } \\
\text { Linearity range: } \\
\text { Telmisartan: } 2-20 \mathrm{mcg} / \mathrm{ml} \\
\text { Hydrochlorthiazide: } 2-12 \mathrm{mcg} / \mathrm{ml} \\
\text { Amlodipine Besylate: }\end{array}$ & [7] \\
\hline
\end{tabular}




\begin{tabular}{|c|c|c|c|c|}
\hline & & & $\begin{array}{l}\text { 10-70mcg/ml } \\
\text { \%RSD: <2 }\end{array}$ & \\
\hline 05 & $\begin{array}{c}\text { Telmisartan and } \\
\text { Amlodipine Besylate }\end{array}$ & $\begin{array}{c}\text { Bulk drugs and } \\
\text { formulations }\end{array}$ & $\begin{array}{c}\text { Detection wave length: } \\
\text { Telmisartan: } 240 \mathrm{~nm} \\
\text { Amlodipine Besylate: } 230 \mathrm{~nm} \\
\text { Linearity range: } \\
\text { Telmisartan: } 0-60 \mu \mathrm{g} / \mathrm{ml} \\
\text { Amlodipine Besylate: } 0-25 \mu \mathrm{g} / \mathrm{ml} \\
\text { Range: } \\
\text { Telmisartan: } 100.57 \% \\
\text { Amlodipine Besylate: } 100.35 \%\end{array}$ & [8] \\
\hline 06 & $\begin{array}{c}\text { Telmisartan } \\
\text { Hydrochlorthiazide and } \\
\text { Amlodipine Besylate }\end{array}$ & $\begin{array}{l}\text { Combined tablet } \\
\text { dosage form }\end{array}$ & $\begin{array}{c}\text { Detection wave length: } \\
\text { First order derivative: } \\
\text { Telmisartan: } 252 \mathrm{~nm} \\
\text { Hydrochlorthiazide: } 271 \mathrm{~nm} \\
\text { Amlodipine Besylate: } 239 \mathrm{~nm} \\
\text { Solvent: Methanol } \\
\text { Linearity range: } 5-30 \mu \mathrm{\mu g} / \mathrm{ml} \\
\text { \%RSD: } \\
\text { Telmisartan: } 0.01292 \\
\text { Hydrochlorthiazide: } 0.03163 \\
\text { Amlodipine Besylate: } \mathbf{0 . 0 2 8 6 3} \\
\end{array}$ & [9] \\
\hline 07 & $\begin{array}{l}\text { Telmisartan and } \\
\text { Metformin } \\
\text { hydrochloride }\end{array}$ & $\begin{array}{c}\text { Combined } \\
\text { pharmaceutical } \\
\text { dosage form }\end{array}$ & $\begin{array}{c}\text { Detection wave length: } \\
\text { Telmisartan: } 217 \mathrm{~nm} \\
\text { Metformin hydrochloride: } 251 \mathrm{~nm} \\
\text { Solvent: Methanol } \\
\text { Linearity range: } 6-16 \mu \mathrm{g} / \mathrm{ml} \\
\% \text { RSD: }<2 \\
\end{array}$ & {$[10]$} \\
\hline
\end{tabular}

Table 2: Methods for the estimation of Telmisartan in single and combination with other drugs by HPLC method

\begin{tabular}{|c|c|c|c|c|}
\hline S. No. & DRUGS & APPLICATION & DESCRIPTION & REF NO \\
\hline 08 & Telmisartan & $\begin{array}{c}\text { Bulk and } \\
\text { pharmaceutical dosage } \\
\text { form }\end{array}$ & $\begin{array}{c}\text { Detection wavelength: } 232 \mathrm{~nm} \\
\text { Column: Welchrom C } 18 \\
\text { Mobile phase: Phosphate buffer pH } 3.3 \text { : } \\
\text { Acetonitrile }(50: 50) \mathrm{v} / \mathrm{v} \\
\text { Flow rate: } 1.0 \mathrm{ml} / \mathrm{min}\end{array}$ & [11] \\
\hline 09 & $\begin{array}{c}\text { Telmisartan and } \\
\text { Metoprolol succinate }\end{array}$ & $\begin{array}{c}\text { Bulk and } \\
\text { pharmaceutical dosage } \\
\text { form }\end{array}$ & $\begin{array}{c}\text { Detection wavelength: } 230 \mathrm{~nm} \\
\text { Column: Reverse phase } \mathrm{C}_{18} \\
\text { (Phenomenex-RP Aqueous) } \\
\text { Mobile phase: } 15 \mathrm{mM} \text { Ammonium acetate } \\
\text { pH 6.5: Acetonitrile(58:24)v/v } \\
\text { Flow rate: } \\
\text { Telmisartan: } 5.3 \mathrm{ml} / \mathrm{min} \\
\text { Metoprolol succinate: } 3.9 \mathrm{ml} / \mathrm{min} \\
\text { Linearity range: } \\
\text { Telmisartan: } 2-10 \mu \mathrm{g} / \mathrm{ml} \\
\text { Metoprolol succinate: } 5-25 \mu \mathrm{g} / \mathrm{ml} \\
\text { Regression coefficient: } 0.9999\end{array}$ & [12] \\
\hline 10 & $\begin{array}{c}\text { Telmisartan } \\
\text { Hydrochlorthiazide } \\
\text { and Amlodipine } \\
\text { Besylate }\end{array}$ & $\begin{array}{c}\text { Bulk and } \\
\text { pharmaceutical dosage } \\
\text { form }\end{array}$ & $\begin{array}{c}\text { Detection wavelength: } 333 \mathrm{~nm} \\
\text { Column: RP-C } 18 \\
\text { Mobile phase: Acetonitrile: Acetate buffer } \\
\text { pH } 5(60: 40 \mathrm{v} / \mathrm{v}) \\
\text { Flow rate: } 1.0 \mathrm{ml} / \mathrm{min} \\
\text { Linearity range: } 20-100 \mu \mathrm{g} / \mathrm{ml} \\
\text { Retention time: } \\
\text { Telmisartan: } 7.10 \mathrm{~min} \\
\text { Hydrochlorthiazide: } 2.56 \mathrm{~min} \\
\text { Amlodipine Besylate: } 3.80 \mathrm{~min} \\
\end{array}$ & [13] \\
\hline 11 & $\begin{array}{l}\text { Telmisartan and } \\
\text { Indapamide }\end{array}$ & $\begin{array}{l}\text { Bulk drug and finished } \\
\text { product }\end{array}$ & $\begin{array}{c}\text { Detection wavelength:240 } \mathrm{nm} \\
\text { Column: Hi Qsil } \mathrm{C}_{18} \\
\text { Mobile phase: Acetonitrile: } 20 \mathrm{mM} \\
\text { Ammonium acetate buffer(pH 4.5) } \\
\text { 60:40v/v } \\
\text { Flow rate:1.0ml/min } \\
\text { Linearity } \mathrm{range:} \\
\text { Telmisartan: } 0.5-60 \mu \mathrm{g} / \mathrm{ml}\end{array}$ & [14] \\
\hline
\end{tabular}




\begin{tabular}{|c|c|c|c|c|}
\hline & & & $\begin{array}{c}\text { Indapamide: } 0.05-2.2 \mu \mathrm{g} / \mathrm{ml} \\
\text { Retention time: } \\
\text { Telmisartan: } 8 \mathrm{~min} \\
\text { Indapamide }: 5 \mathrm{~min}\end{array}$ & \\
\hline 12 & $\begin{array}{c}\text { Telmisartan and } \\
\text { Ramipril }\end{array}$ & $\begin{array}{l}\text { Pharmaceutical } \\
\text { formulations }\end{array}$ & $\begin{array}{c}\text { Detection wavelength: } 210 \mathrm{~nm} \\
\text { Column: Inertsil ODS } \mathrm{C}_{18} \\
\text { Mobile phase: Potassiun dihydrogen } \\
\text { phosphate buffer(pH } 2.8): \text { Acetonitrile } \\
60: 40 \mathrm{v} / \mathrm{v} \\
\text { Flow rate: } 1.5 \mathrm{ml} / \mathrm{min} \\
\text { Linearity range: } \\
\text { Telmisartan: } 2.5-25.5 \mathrm{ppm} \\
\text { Ramipril: } 3-7.25 \mathrm{ppm} \\
\text { Retention time: } \\
\text { Telmisartan: } 5.7 \mathrm{~min} \\
\text { Ramipril: } 10.8 \mathrm{mins}\end{array}$ & [15] \\
\hline 13 & $\begin{array}{c}\text { Telmisartan and } \\
\text { Hydrochlorothaizide }\end{array}$ & $\begin{array}{l}\text { Pharmaceutical dosage } \\
\text { form }\end{array}$ & $\begin{array}{c}\text { Detection wavelength: } 230 \mathrm{~nm} \\
\text { Column: Hypersil BDS C-18 } \\
\text { Mobile phase: Tetrabutyl ammonium } \\
\text { hydroxide phosphate buffer(pH } \\
\text { 2.26):Acetonitrile } 78: 22 \mathrm{v} / \mathrm{v} \\
\text { Column temperature: } 30^{0} \mathrm{C} \\
\text { Linearity range: } \\
\text { Telmisartan: } 5-200 \mu \mathrm{g} / \mathrm{ml} \\
\text { Hydrochlorothaizide:1.6-64 } \mu \mathrm{g} / \mathrm{ml}\end{array}$ & [16] \\
\hline 14 & $\begin{array}{l}\text { Telmisartan and } \\
\text { Pioglitazone }\end{array}$ & $\begin{array}{c}\text { Bulk and } \\
\text { pharmaceutical dosage } \\
\text { form }\end{array}$ & $\begin{array}{c}\text { Detection wavelength: } 210 \mathrm{~nm} \\
\text { Column: Phenomenex } \mathrm{C}_{8} \\
\text { Mobile phase: Acetonitrile: Ammonium } \\
\text { dihydrogen phosphate( } \mathrm{pH} 4.8) 65: 35 \mathrm{v} / \mathrm{v} \\
\text { Flow rate: } 1.0 \mathrm{ml} / \mathrm{min} \\
\text { Linearity range: } \\
\text { Telmisartan: } 10-50 \mu \mathrm{g} / \mathrm{ml} \\
\text { Pioglitazone: } 7.5-37.5 \mu \mathrm{g} / \mathrm{ml} \\
\text { Retention time: } \\
\text { Telmisartan: } 2.38 \mathrm{~min} \\
\text { Pioglitazone: } 3.16 \mathrm{~min}\end{array}$ & [17] \\
\hline 15 & $\begin{array}{l}\text { Telmisartan and } \\
\text { Ramipril }\end{array}$ & Tablet formulation & $\begin{array}{c}\text { Detection wavelength: } 210 \mathrm{~nm} \\
\text { Column: Genesis } \mathrm{C}_{18} \\
\text { Mobile phase: } 0.01 \mathrm{M} \text { potassium } \\
\text { dihydrogen phosphate buffer }(\mathrm{pH} 3.4): \\
\text { Methanol: Acetonitrile } 15: 15: 75 \mathrm{v} / \mathrm{v} \\
\text { Flow rate: } 1.0 \mathrm{ml} / \mathrm{min} \\
\text { Linearity } \mathrm{range}: \\
\text { Telmisartan: } 28-52 \mu \mathrm{g} / \mathrm{ml} \\
\text { Ramipril: } 3.5-6.5 \mu \mathrm{g} / \mathrm{ml} \\
\text { Retention time: } \\
\text { Telmisartan: } 4.98 \mathrm{~min} \\
\text { Ramipril: } 3.68 \mathrm{~min}\end{array}$ & [18] \\
\hline 16 & Telmisartan & Tablet formulation & $\begin{array}{c}\text { Detection wavelength: } 256 \mathrm{~nm} \\
\text { Column: Chromosil } \mathrm{C}_{18} \\
\text { Mobile phase: Methanol:0.1\% O- } \\
\text { phoshoric acid : Acetonitrile } \\
\text { 80:0.5:13v/v/v } \\
\text { Flow rate }: 1.5 \mathrm{ml} / \mathrm{min} \\
\text { Retention time: } 2.7 \mathrm{~min}\end{array}$ & [19] \\
\hline 17 & $\begin{array}{c}\text { Telmisartan and } \\
\text { Amlodipine Besylate }\end{array}$ & Combined dosage form & $\begin{array}{c}\text { Detection wavelength: } 250 \mathrm{~nm} \\
\text { Column: Prontosil- } \mathrm{C}_{18} \\
\text { Mobile phase: Methanol: potassium } \\
\text { dihydrogen phosphate buffer (pH 4) 75: } \\
\text { 25v/v } \\
\text { Flow rate: } 0.7 \mathrm{ml} / \mathrm{min} \\
\text { Linearity range: } \\
\text { Telmisartan: } 1-35 \mu \mathrm{g} / \mathrm{ml} \\
\text { Amlodipine Besylate: } 2-16 \mu \mathrm{g} / \mathrm{ml} \\
\text { Retention time: } \\
\text { Telmisartan: } 8.2 \mathrm{~min}\end{array}$ & [20] \\
\hline
\end{tabular}




\begin{tabular}{|c|c|c|c|c|}
\hline & & & Amlodipine Besylate: 3.5 min & \\
\hline 18 & Telmisartan & $\begin{array}{l}\text { Bulk drug and tablet } \\
\text { formulation }\end{array}$ & $\begin{array}{c}\text { Detection wavelength: } 220 \mathrm{~nm} \\
\text { Column: Symetry C-18 } \\
\text { Mobile phase: } 10 \mathrm{mM} \text { Ammonium acetate } \\
\text { buffer(pH 4) : Acetonitrile 40:60v/v } \\
\text { Flow rate: } 1.0 \mathrm{ml} / \mathrm{min} \\
\text { Linearity range: } 1-16 \mu \mathrm{g} / \mathrm{ml} \\
\end{array}$ & [21] \\
\hline 19 & $\begin{array}{c}\text { Telmisartan and } \\
\text { Metoprolol succinate }\end{array}$ & $\begin{array}{l}\text { Bulk drug and } \\
\text { formulation }\end{array}$ & $\begin{array}{c}\text { Detection wavelength: } 223 \mathrm{~nm} \\
\text { Column: Hi Q Sil } \mathrm{C}_{18} \\
\text { Mobile phase: Methanol: } 10 \mathrm{mM} \\
\text { potassium dihydrogen phosphate buffer: } \\
\text { 10mM Hexane sulphonic acid: } \\
\text { 18:10:10v/v/v } \\
\text { Flow rate: } 1.0 \mathrm{ml} / \mathrm{min} \\
\text { Linearity range: } \\
\text { Telmisartan: } 5-60 \mu \mathrm{g} / \mathrm{ml} \\
\text { Metoprolol succinate: } 5-80 \mu \mathrm{g} / \mathrm{ml} \\
\text { Retention time: } \\
\text { Telmisartan: } 5.653 \mathrm{~min} \\
\text { Metoprolol succinate: } 3.067 \mathrm{~min}\end{array}$ & [22] \\
\hline 20 & $\begin{array}{l}\text { Telmisartan and } \\
\text { Chlorthalidone }\end{array}$ & $\begin{array}{l}\text { Bulk and tablet } \\
\text { formulation }\end{array}$ & $\begin{array}{c}\text { Detection wavelength: } 242 \mathrm{~nm} \\
\text { Column: } \mathrm{C}_{18} \\
\text { Mobile phase: Acetonitrile: Methanol } \\
\text { 85:15v/v } \\
\text { Flow rate: } 1.0 \mathrm{ml} / \mathrm{min} \\
\text { Linearity range: } \\
\text { Telmisartan: } 15-56 \mu \mathrm{g} / \mathrm{ml} \\
\text { Chlorthalidone: } 5-17.5 \mu \mathrm{g} / \mathrm{ml} \\
\text { Retention time: } \\
\text { Telmisartan: } 3.96 \mathrm{~min} \\
\text { Chlorthalidone: } 2.63 \mathrm{~min}\end{array}$ & [23] \\
\hline 21 & $\begin{array}{c}\text { Telmisartan and } \\
\text { Atorvastatin calcium }\end{array}$ & $\begin{array}{c}\text { API and tablet dosage } \\
\text { form }\end{array}$ & $\begin{array}{c}\text { Detection wavelength: } 235 \mathrm{~nm} \\
\text { Column: Boston ODS } \mathrm{C}_{18} \\
\text { Mobile phase: Methanol: Acetonitrile: } \\
\text { Buffer } 35: 25: 40 \\
\text { Flow rate: } 1.0 \mathrm{ml} / \mathrm{min} \\
\text { Retention time: } \\
\text { Telmisartan: } 3.490 \mathrm{~min} \\
\text { Atorvastatin calcium }: 2.350 \mathrm{~min}\end{array}$ & [24] \\
\hline 22 & $\begin{array}{c}\text { Telmisartan and } \\
\text { Amlodipine Besylate }\end{array}$ & $\begin{array}{l}\text { Pharmaceutical dosage } \\
\text { form }\end{array}$ & $\begin{array}{c}\text { Detection wavelength: } 201 \mathrm{~nm} \\
\text { Column: Luna } \mathrm{C}_{18} \\
\text { Mobile phase: Phosphate buffer(pH 3): } \\
\text { Acetonitrile: } 60: 40 \mathrm{v} / \mathrm{v} \\
\text { Flow rate: } 1.0 \mathrm{ml} / \mathrm{min} \\
\text { Linearity range: } \\
\text { Telmisartan: } 160-240 \mu \mathrm{g} / \mathrm{ml} \\
\text { Amlodipine Besylate: } 25-45 \mu \mathrm{g} / \mathrm{ml} \\
\text { Retention time: } \\
\text { Telmisartan: } 4.427 \mathrm{~min} \\
\text { Amlodipine Besylate: } 2.643 \mathrm{~min}\end{array}$ & [25] \\
\hline
\end{tabular}

Table 3: Methods for the estimation of Telmisartan in single and combination with other drugs by HPTLC method

\begin{tabular}{|c|c|c|c|c|}
\hline S. No. & DRUGS & APPLICATION & DESCRIPTION & REF NO \\
\hline 23 & $\begin{array}{r}\text { Telmisartan and } \\
\text { Amlodipine Besylate }\end{array}$ & $\begin{array}{l}\text { Tablet dosage } \\
\text { form }\end{array}$ & $\begin{array}{c}\text { Column: Aluminium packed silica } \\
\text { gel 60F 254 } \\
\text { Mobile phase: Ethyl acetate: } \\
\text { Methanol: Ammonia: Glacial } \\
\text { acetic acid 7.5:1.5:1:0.2v/v/v/v } \\
\text { Detection of spot: } 226 \mathrm{~nm} \\
\text { Retardation Factor: } \\
\text { Telmisartan: } 0.60 \\
\text { Amlodipine Besylate }: 0.34\end{array}$ & {$[26]$} \\
\hline 24 & $\begin{array}{l}\text { Telmisartan and } \\
\text { Indapamide }\end{array}$ & $\begin{array}{l}\text { Pharmaceutical } \\
\text { dosage form }\end{array}$ & $\begin{array}{l}\text { Column: Precoated Silica gel GF } \\
254 \\
\text { Mobile phase: Hexane: Ethyl } \\
\text { acetate: Methanol: Glacial acetic }\end{array}$ & [27] \\
\hline
\end{tabular}




\begin{tabular}{|l|c|c|}
\hline & & acid 14:6:2:1 \\
& & Linearity range: Telmisartan: \\
& & $2000-7000 \mathrm{ng} / \mathrm{spot}$ \\
& Indapamide: \\
& & $75-262.5 \mathrm{ng} / \mathrm{spot}$ \\
& Retardation factor: \\
& & Telmisartan: 0.21 \\
\hline
\end{tabular}

\section{CONCLUSION}

This review discussed the reported spectroscopic techniques like UV Spectroscopy and Chromatographic techniques like HPLC, HPTLC methods developed and validated for the estimation of Telmisartan in single and in combination with other pharmaceutical drugs. It can be reviewed that out of all these techniques HPLC with UV detection was extensively with best suitable solvents like acetonitrile, potassium dihydrogen phosphate buffer, methanol, ammonium acetate buffer for better resolution. It was also observed that a flow rate of $1.0 \mathrm{ml} / \mathrm{min}$ gives best compounds detection. Hence all these methods were simple, precise, accurate, and offers reproducibility with reliability with low in cost in compared with advanced technology.

\section{ACKNOWLEDGEMENTS}

I am very grateful to Chalapathi Institute of Pharmaceutical Sciences, Lam, Guntur, for providing support, guidance, and facilities.

\section{REFERENCES}

[1] Nerenberg KA, Zarnke KB, Leung AA, Dasgupta K, Butalia S, McBrien

K, Hypertension Canada's 2018. Guidelines for Diagnosis, Risk
Assessment, Prevention, and Treatment of Hypertension in Adults and Children, Canadian. J. Cardiology, 34(5), 2018, 506-525.

[2] Bakheit A, Abd-Elgalil A, Telmisartan, Profiles of Drug Substances, Excipients and Related Methodology, 40, 2015, 371-429.

[3] Patel N, Jayvadan K Patel, Analytical Methodologies for Determination of Telmisartan: an Overview, Int. J. Pharmacy and Pharma.Sci, 5(1), 2013, 17-22.

[4] Patel K, Dhudasia K, Patel A, Dave J, Stress Degradation Studies on Telmisartan and Development of a Validated Method by UV Spectrophotometry in Bulk and Pharmaceutical Dosage Forms, Pharma.Methods, 2(4), 2011, 253259.

[5] Patel K, Patel A, Dave J, Absorbance Correction Method for Estimation of Telmisartan and Metoprolol Succinate in Combined Tablet Dosage Forms, Pharma.Methods, 3(2), 2012, 106-111.

[6] Rathod SD, Patil P, Waghmare SS, Chaudhari P,UV-Spectrophotometric 
Method for Estimation of Telmisartan in Bulk and Tablet Dosage Form, Int.

J. Pharma. Sci. Res, 3(10), 2012, 3936-3939.

[7] Sasidhar R, Vidyadhara S, Deepti B, Tejaswi K, A validated UV Spectrophotometric Method for the Simultaneous Estimation of Hydrochlorothiazide, Amlodipine Besylate and Telmisartan in Bulk and Combined Tablet Dosage Form, Der. Pharmacia. Lettre, 6(4), 2014, 449455.

[8] Sonia K, Nappinnai M, Panneerselvam P, Stability Indicating UV Method for Simultaneous Determination of Telmisartan and Amlodipine Besylate, Res. J. Pharmacy and Tech, 11(11), 2018, 4825-4828.

[9] Raskar M, Godge G, Chitale A, Validated Simultaneous Spectrophotometric Estimation of Telmisartan, Hydrochlorthiazide and Amlodipine Besylate in Combined Tablet Dosage Form, Der. Pharmacia. Lettre, 7(11), 2015, 220-224.

[10] Khristi A, Baraka J, Dasvani B, Analytical Method Development and Validation of First Order Derivative Spectrophotometric Method for Simultaneous Estimation of Telmisartan and Metformin Hydrochloride in their Combined
Pharmaceutical Dosage Form, Int. J. Pharma. Qua. Assurance, 11(1), 2020, 60-68.

[11] Ravisankar P, Vineela C, Koushik O, Novel Simultaneous Separation and Quantitative Determination of Telmisartan, Losartan, Olmesartan and Irbesartan in presence of Hydrochlorothiazide by Isocratic RPHPLC, Adv. J. Pharma. Life. Sci. Res, 3, 2015, 1-18.

[12] Anusha M, Bharathi D, Simultaneous Estimation of Metoprolol Succinate and Telmisartan in Bulk and Pharmaceutical Dosage Forms by RP-HPLC, Int. J. Pharma. Sci. Rev and Res, 16(2), 2012, 111-115.

[13] Sasidhar R, Vidyadhara S, Method for the Simultaneous Determination of Hydrochlorothiazide, Amlodipine Besylate and Telmisartan in Bulk and Pharmaceutical Formulation, Oeriantal. J. Chem, 30(4), 2014, 1815-1822.

[14] Satpute C, Pagare P, Jadha V, Simultaneous Estimation of Telmisartan and Indapamide in Bulk Drug and Finished products by RPHPLC method, Res. J. Pharm and Tech, 6(11), 2013, 1217-1224.

[15] Jawla S, Jeyalakshmi K, Development and Validation of Simultaneous HPLC Method for Estimation of Telmisartan and Ramipril in Pharmaceutical Formulations, Int. J. 
Pharm. Tech and Res, 2(2), 2010, 1625-1633.

[16] Shi Q, Chen Q, Wang P, Zhao L, Cao $\mathrm{Y}$, Determination of Telmisartan and Hydrochlorothiazide in Compound Telmisartan Capsules by HPLC, Zhongyuo Yiyao Gongye Zazhi, 36(3), 2005, 165-166.

[17] PremAnand D, Senthilkumar K, A new RP-HPLC Method Development and Validation for Simultaneous Estimation of Telmisartan and Pioglitazone in Pharmaceutical Dosage Form, Int. J. Chem. Tech. Res, 3(1), 2011, 448-454.

[18] Kurade V, Pai M, Gude R, RP-HPLC Estimation of Ramipril and Telmisartan in Tablets, Ind. $J$. Pharma. Sci, 71(2), 2009, 148-151.

[19] Rao M, Nagendrakumar A, Shivnadh M, Validated RP-HPLC Method for the Estimation of Telmisartan in Tablet Formulation, Bulletin.

Pharma. Res, 2(2), 2012, 50-55.

[20] Fatatry H, Habib A, Sherin F H, Development and Validation of Quality by Design Optimized Reversed phase HPLC Method for Simultaneous Estimation of Telmisartan and Amlodipine, Int. J. Pharma. Res, 4(10), 2013, 697-701.

[21] Alhazmi HA, Alnami AM, Arishi MAA, Alameer RK, Al Bratty M, Ur Rehman Z, A Fast and Validated
Reversed-Phase HPLC Method for Simultaneous Determination of Simvastatin, Atorvastatin, Telmisartan and Irbesartan in Bulk Drugs and Tablet Formulations, Scientia Pharmaceutica, 86(1), 2017, 1-13.

[22] Mahaparale SP, Gonjari ID, Jayaveera KN, Stability Indicating HPLC Method for Simultaneous Estimation of Metoprolol Succinate and Telmisartan, J.Liq.Chrom and Rel.Tech, 36(18), 2013, 2601-2611.

[23] Parmar K, Patel N, Stability indicating RP-HPLC Method for Simultaneous Determination of Telmisartan and Chlorthalidone in Bulk and Pharmaceutical Dosage Form, Int. J. Pharma. Tech. Res, 5(4), 2013, 1728-1735.

[24] Bangaruthalli J, Harini U, Simultaneous Estimation of Telmisartan and Atorvastatin Calcium in API and Tablet Dosage Form, J.Drug.Del and Therap, 9(1), 2019, 175-179.

[25] Chabukswar A, Jagdale S, Kumbhar S, HPLC Method Development for Telmisartan and Amlodipine, Res. J. Pharmacy and Tech, 3(4), 2010, 1227-1230.

[26] Thomas A.B, Jagdale S N, Nanda R K, Kothapalli L P, Deshpande A D, Stability Indicating Hptlc Method for the Simultaneous Determination of Amlodipine Besylate and Telmisartan 
from Tablet Dosage Form,

J.Pharma.Res, 10(2), 2011, 66-72.

[27] Sureja D, Parmar G, Patel M, Vadalia $\mathrm{K}$, Development and Validation of HPTLC Method for Simultaneous
Estimation of Telmisartan and Indapamide in Pharmaceutical Solid Dosage Form, J. Chem and Pharma. Res, 7(11), 2015, 236-240. 